\title{
OPTIMIZATION OF GROUNDWATER RESOURCES MANAGEMENT IN POLLUTED AQUIFERS
}

\author{
K.L. KATSIFARAKIS* \\ M. MOUTI \\ K. NTROGKOULI
}

Received: 16/09/08

Accepted: 09/02/09

\author{
Division of Hydraulics and Environmental Engineering, \\ Dept. of Civil Engineering, A.U.Th, \\ GR- 54124 Thessaloniki, Macedonia, Greece
}

*to whom all correspondence should be addressed: e-mail: klkats@civil.auth.gr

\begin{abstract}
This paper focuses on optimal management of aquifers with pollution problems. Genetic algorithms are used as the optimization tool, while advective pollutant transport is simulated by means of a moving point technique. The approach is illustrated by means of two application examples. In the first we seek the maximum total clean water pumping rate $Q_{S}$ from three production wells, situated in the same restricted area with two wells that inject polluted water. Results show that $Q_{\mathrm{s}}$ depends heavily on the dimensions of the available area. In the second application example, the total flow rate of the production wells is fixed and the minimum treatment cost of pumped water is sought. Best solutions resulting from a number of runs are classified in 3 different patterns, which are comparatively evaluated. Some remarks on taking into account dispersive mass transport conclude the paper.
\end{abstract}

KEYWORDS: aquifer pollution, optimal management, genetic algorithms, moving points, penalty function.

\section{INTRODUCTION}

Fresh water availability is a basic prerequisite for development of human activities. Water shortages occur quite often in many areas of the world, calling for optimal management of both surface and groundwater resources. The latter are more abundant at the global scale. Moreover, their quality is usually better, since they are naturally more protected. It should be mentioned though, that, once polluted, their restoration is more difficult. For these reasons, protection and restoration of groundwater resources is a very challenging task, including several stages, such as identification of possible pollution sources, field measurements, mathematical and numerical modeling of mass transport in different aquifer types and optimization of control measures and techniques.

Due to the importance of groundwater resources, scientific literature on their protection, restoration and management is very rich, e.g. Bear and Bachmat (1990), Bedient et al (1994), Katsifarakis (2000), Voudrias (2001). In this paper two problems of optimal development of a polluted aquifer are discussed. In both of them, polluted water is injected to the aquifer at constant rate, through two wells. The first optimization task is to find the maximum total flow rate $Q_{s}$ that can be safely pumped from a system of 3 wells, located in a predefined area, including the injection wells, too. In the second problem the total flow rate $Q_{s}$, pumped from the 3 wells is fixed and the task is to minimize the water treatment cost, for a 3-year period. In both cases, injected water is considered as safe, if it remains in the aquifer for more than 2 years.

To tackle such problems, a flow simulation model, which provides groundwater velocities, should be combined with an optimization tool. Genetic algorithms, which are particularly efficient in optimization problems, when the respective objective functions exhibit many local optima or discontinuous derivatives, have been adopted in this paper. 


\section{OUTLINE OF THE OPTIMIZATION TOOL}

Scientific literature on genetic algorithms, which were initially introduced by Holland (1975), is quite rich, e.g. Goldberg (1989), Michalewicz (1996), Reeves and Raw (2003). In brief, they are essentially a mathematical imitation of a biological process, namely that of evolution of species. They start with a number of random potential solutions of the problem (in our case pumping schemes). These solutions, which are called chromosomes, constitute the population of the first generation. In binary genetic algorithms, each chromosome is a binary string. The number of its characters, which are called genes, is usually predetermined.

Then a fitness value is assigned to each chromosome of the first generation, by means of a pertinent evaluation function or process. This process depends entirely on the specific application of genetic algorithms and may include a penalty function, to reduce chromosome's fitness, if the respective solution violates any constraint of the problem. In both cases discussed in this paper, the evaluation process includes the flow and mass transport simulation model.

When evaluation of every chromosome is completed, the next generation is produced, through application of certain operators, which imitate biological processes. The main genetic operators are: a) selection b) crossover and c) mutation. Many other operators have been also proposed and used.

Selection is used first. It can be accomplished in many ways. In our code the tournament method has been preferred. Selection leads to an intermediate population, in which better chromosomes have, statistically, more copies. These copies substitute some of the worst chromosomes. Moreover the best chromosome of each generation is separately preserved through the selection process (elitist approach). Then, the rest of the operators apply to randomly selected members of this intermediate population. In our code typical crossover and mutation, together with antimetathesis, an operator described by Katsifarakis and Karpouzos (1998) and Katsifarakis et al (1999a), are used. The result is an equal number of new chromosomes, i.e. new solutions, which replace the old ones. Thus, the next generation is formed.

The whole process, i.e. evaluation-selection-crossover-mutation-antimetathesis, is repeated for a number of generations, depending mainly on computational load per chromosome evaluation and available computer resources. It is anticipated that, at least in the last generation, a chromosome will prevail, which represents a sub-optimal (if not the global optimal) solution of the problem. Identification of a number of very good solutions is an asset, too.

\section{SIMULATION OF FLOW AND MASS TRANSPORT}

It has already been mentioned that simulation of flow and mass transport is an essential part of the chromosome evaluation procedure. Actually, it is used to check whether the pumping scheme, represented by a particular chromosome, results in pollution of the water, that arrives at the production wells. The respective calculations, repeated for every chromosome of each generation, form the larger part of the total computational volume. For this reason they should be reduced as much as possible. We assume that groundwater flow takes place in a horizontally infinite, homogeneous, confined aquifer. Then, use of the continuity equation, together with Darcy's law and the superposition principle, leads to the following expressions for water velocities $V_{x}, V_{y}$ at any point $(x, y)$ :

$$
\begin{aligned}
& V_{x}=-\frac{1}{2 \pi \theta a_{a q}} \sum_{i=1}^{W T} Q_{i} \frac{x-x_{i}}{\left(x-x_{i}\right)^{2}+\left(y-y_{i}\right)^{2}} \\
& V_{y}=-\frac{1}{2 \pi \theta a_{a q}} \sum_{i=1}^{W T} Q_{i} \frac{y-y_{i}}{\left(x-x_{i}\right)^{2}+\left(y-y_{i}\right)^{2}}
\end{aligned}
$$

In these equations, $a_{a q}$ and $\theta$ are aquifer's width and effective porosity, respectively, $x_{i}, y_{i}$ the coordinates of well $i$ and $Q_{i}$ its flow rate, while WT is the total number of wells (WT=5 in our case). Water velocities serve to simulate advective mass transport, by means of a moving point technique, in the following way: Points that represent polluted water entering the flow field, are initially placed at the perimeter of a circle around each injection well. Then, local water velocities are calculated at the location of each point, by means of equations (1) and 
(2). The coordinates $x_{i n}, y_{\text {in }}$ of the new position of each point after a time period $\Delta T$, are derived using the following relationships:

$x_{\text {in }}=x_{\text {io }}+V_{x} \cdot \Delta T$

$\mathrm{y}_{\text {in }}=\mathrm{y}_{\text {io }}+\mathrm{V}_{\mathrm{y}} \cdot \Delta \mathrm{T}$

where $x_{i o}, y_{i o}$ are the coordinates of the previous moving point position. These calculations are repeated for a predetermined number of time-steps $\Delta \mathrm{T}$, covering the period of pollutant deactivation ( 2 years in our case). It should be mentioned that points are tracked until the last time-step, except when they arrive at a production well W. A point $P$ is assumed to arrive at W, when one of the following conditions holds (Katsifarakis et al, 1999b): 1) Distance between $\mathrm{P}$ and $\mathrm{W}$ is smaller than a distance $r_{\mathrm{w}}$, depending on the flow rate of $\mathrm{W}$ and the size of the time-step and 2) Location of $P$ at the end of a time-step is closer to $W$ than to its previous location and the distance between $\mathrm{P}$ and $\mathrm{W}$ is smaller than $4 \cdot \mathrm{r}_{\mathrm{w}}$.

\section{APPLICATIONS}

The basic common features of the two optimization problems, discussed in this paper, are the following: Polluted water is injected to the aquifer, through wells $B$ and $C$ (shown in figures 1 to 5), at constant rates 40 and $50 \mathrm{I} \mathrm{s}^{-1}$ respectively. Their coordinates are $(300,800)$ and $(800$, $400)$ respectively, while those of production well $A$ are $(300,0)$. Two more production wells will be constructed in a predefined area, which includes the aforementioned wells $A, B$ and $C$.

The first optimization task is to find the maximum total flow rate $Q_{s}$ that can be safely pumped from the system of the 3 production wells. As stated earlier, we assume that injected pollutants are completely deactivated, if they stay inside the aquifer for more than 2 years.

Actually our task is to find the flow rates of all 3 production wells, together with the best locations for the 2 of them. Then each chromosome represents a set of 2 pairs of coordinates and 3 well flow rates $Q_{i}$, expressed as binary numbers. Its length is calculated on the basis of QMAX, namely the maximum anticipated flow rate per well and the dimensions of the area, which is available for well construction.

The fitness value of each chromosome is given as

$\mathrm{VB}=\sum_{\mathrm{i}=1}^{3} \mathrm{Q}_{\mathrm{i}}-\mathrm{PEN}$

where production well flow rates are expressed in $1 \mathrm{~s}^{-1}$ and PEN is a penalty, imposed if polluted water (namely moving points) arrive at any production well, in less than 2 years. So, calculation of PEN requires solution of the flow simulation model, for the combination of well locations and flow rates, represented by the chromosome. We have chosen the following form for the term PEN:

$\mathrm{PEN}=\sum\left(100+10\left(\mathrm{TT} 1-\mathrm{Tb}_{\mathrm{i}}-1\right)\right.$

where TT1 is the total number of time steps (covering the 2 year period) and $\mathrm{Tb}_{\mathrm{i}}$ is the time step at which the moving point $i$ arrived at a production well. Of course summation extends to moving points with $\mathrm{Tb}_{\mathrm{i}}<\mathrm{TT} 1$ only. Thus PEN depends both on the number of the violated constraints (number of moving points arriving at production wells) and the magnitude of the violation (time step of moving point arrival).

In the second problem the required total flow rate $Q_{s}$, pumped from the 3 production wells is fixed to $600 \mathrm{I} \mathrm{s}^{-1}$ and the task is to minimize a simple function, representative of water treatment cost for a 3-year period, which serves for chromosome evaluation and has the following form:

$\mathrm{VB}=\sum\left(1000+\mathrm{Q}_{\mathrm{j}}\left(\mathrm{TT} 2-\mathrm{Tb}_{\mathrm{i}}-1\right)\right)$

where TT2 is the number of time-steps (covering the 3 year period) and $Q_{j}$ is the flow rate of well $j$, at which moving point $i$ arrives. Of course summation extends to moving points with $\mathrm{Tb}_{\mathrm{i}}$ $<$ TT1 only. The idea here is that the pollution load is attenuated as its residence time inside the aquifer increases. The chromosome form is the same with that of the first problem. Well flow rates have to be "repaired", though, in order to fix their sum to $600 \mathrm{I} \mathrm{s}^{-1}$. 


\subsection{Results}

The code which has been constructed for each problem has been run several times, using the following set of parameters for the genetic algorithm code: population size PS $=100$, number of generations $N G=1000$, crossover probability $C R P=0.40$, selection constant $K K=3$, while mutation/antimetathesis probability is approximately equal to the inverse of the chromosome length.

Typical best results for the first problem appear in Tables 1 and 2. The dimensions of the area available for the construction of the new wells are both equal to $1500 \mathrm{~m}$ for the first set and $1000 \mathrm{~m}$ for the second one. It can be seen from Table 1 that results of 3 runs $(1,4$ and 5$)$ are practically the same. Run 2, corresponds to a different well layout, which is shown in Fig. 1, but the difference in $Q_{s}$ is small. Run 3 is obviously a failure. It can also be seen from Table 1 , that $Q_{s}$ could be even larger, if we allowed flow rates larger than $400 \mathrm{I} \mathrm{s}^{-1}$ from a single well.

As shown in Table 2, maximum safe total flow rate is reduced drastically, when the dimensions of the area available for the construction of the new wells is $1000 \times 1000$ only. The well layout of run 5 , which is practically the same with that of run 2, appears in Figure 2. In all cases a new well is placed close to the vertex of the available area, which is the most distant from the existing production well.

Table 1. Typical best results for the first problem (area $1500 \times 1500$ )

\begin{tabular}{ccccccc}
\hline Run & $\mathbf{Q}_{\mathbf{A}}$ & $\mathbf{Q}_{\mathbf{D}}$ & $\mathbf{X}_{\mathbf{D}}, \mathbf{Y}_{\mathrm{D}}$ & $\mathbf{Q}_{\mathrm{E}}$ & $\mathbf{X}_{\mathrm{E}}, \mathbf{Y}_{\mathrm{E}}$ & $\mathbf{Q}_{\mathbf{s}}$ \\
\hline $\mathbf{1}$ & 174.6 & 199.6 & 45,1498 & 400 & 1413,1313 & 774.2 \\
\hline $\mathbf{2}$ & 199.6 & 199.6 & 1479,7 & 400 & 1375,1294 & 799.2 \\
\hline $\mathbf{3}$ & 99.4 & 400 & 1170,1461 & 99.4 & 29,234 & 598.8 \\
\hline $\mathbf{4}$ & 174.6 & 400 & 1403,1314 & 199.6 & 29,1496 & 774.2 \\
\hline $\mathbf{5}$ & 174.6 & 199.6 & 48,1499 & 400 & 1416,1313 & 774.2 \\
\hline
\end{tabular}

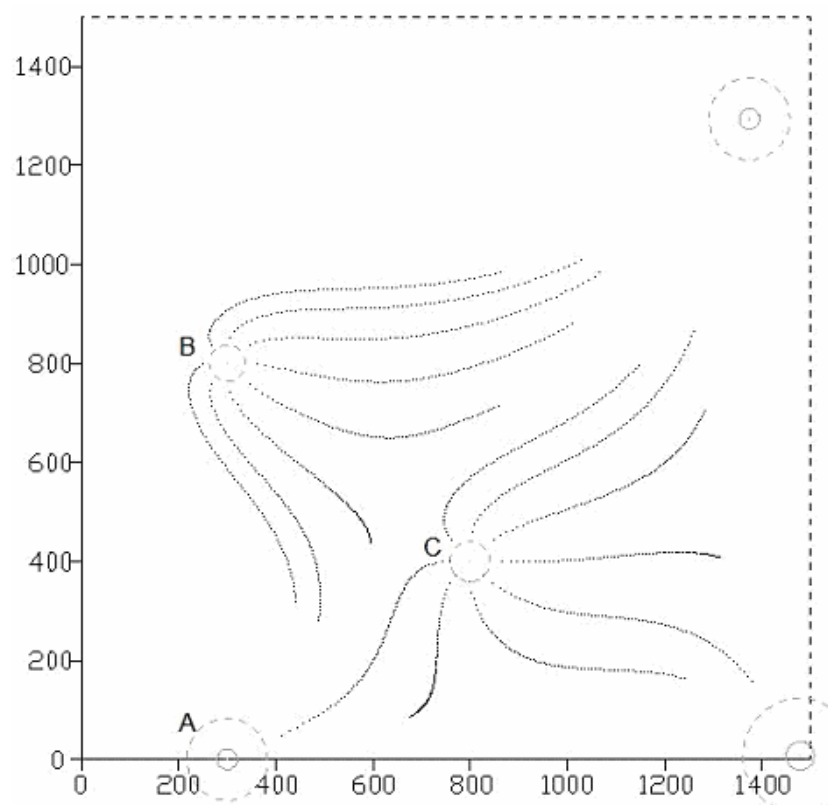

Figure 1. Typical best results (first problem, area 1500x1500)

Table 2. Typical best results for the first problem (area $1000 \times 1000$ )

\begin{tabular}{ccccccc}
\hline Run & $\mathbf{Q}_{\mathbf{A}}$ & $\mathbf{Q}_{\mathrm{D}}$ & $\mathbf{X}_{\mathrm{D}}, \mathbf{Y}_{\mathrm{D}}$ & $\mathbf{Q}_{\mathrm{E}}$ & $\mathbf{X}_{\mathrm{E}}, \mathbf{Y}_{\mathrm{E}}$ & $\mathbf{Q}_{\mathbf{s}}$ \\
\hline $\mathbf{1}$ & 31.1 & 74.6 & 928,962 & 149.7 & 2,53 & 255.4 \\
\hline $\mathbf{2}$ & 51.1 & 74.6 & 0,215 & 74.6 & 857,998 & 200.2 \\
\hline $\mathbf{3}$ & 149.7 & 74.6 & 923,929 & 27.0 & 773,374 & 251.3 \\
\hline $\mathbf{4}$ & 55.8 & 74.6 & 964,978 & 55.8 & 13,247 & 186.1 \\
\hline $\mathbf{5}$ & 51.1 & 74.6 & 3,215 & 74.6 & 878,990 & 200.2 \\
\hline
\end{tabular}




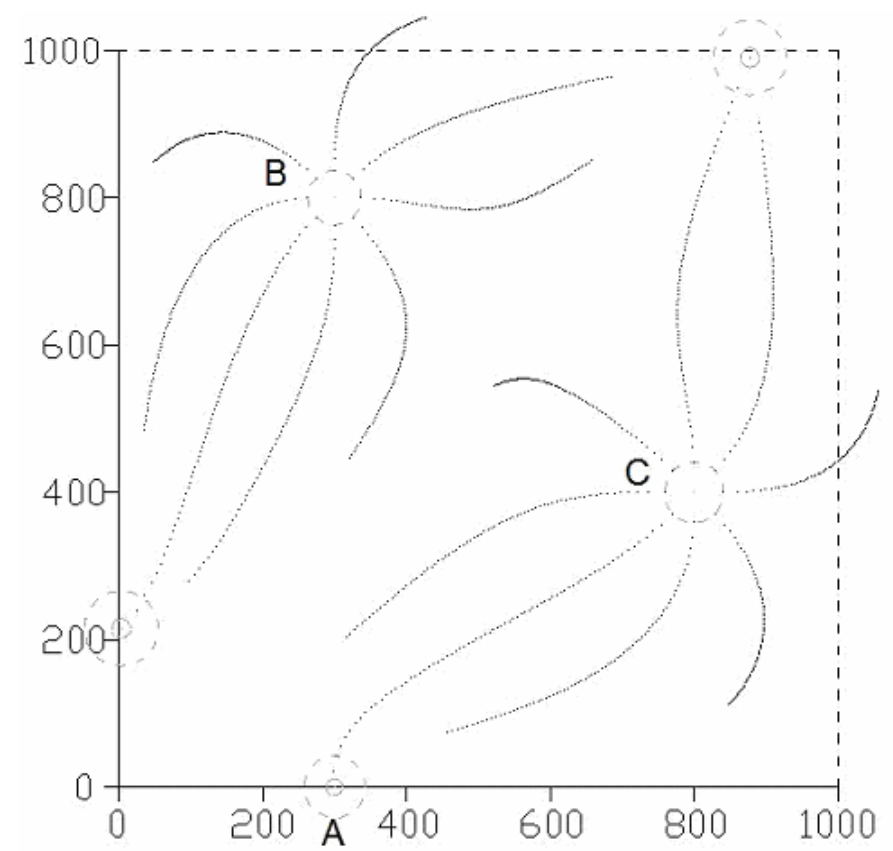

Figure 2. Typical best results (first problem, area 1000x1000)

Typical best results for the second problem, summarized in Table 3, can be classified in 3 different patterns which appear in Figures 3, 4, and 5. In the first (Fig. 3), which corresponds to runs 3, 4 and 6 and leads generally to smaller "cost" values, a production well with low flow rate is placed close to injection well $C$. Thus it traps most of the polluted water. In the second pattern (Fig. 4), which corresponds to runs 1 and 2, a production well with low flow rate is placed close to well $A$, thus protecting it from pollution. In the third pattern, representing runs 5 and 7 and shown in Fig. 5, new production wells are placed away from the existing wells. This is what we anticipated. Solutions following the first pattern are actually not that good, since pollutants injected in well $C$, have very small residence time inside the aquifer, and the respective pollutant attenuation is very small. Pattern $B$ is more interesting, since it allows for pollutant attenuation. It should be further checked, though, if well $A$ is thoroughly protected.

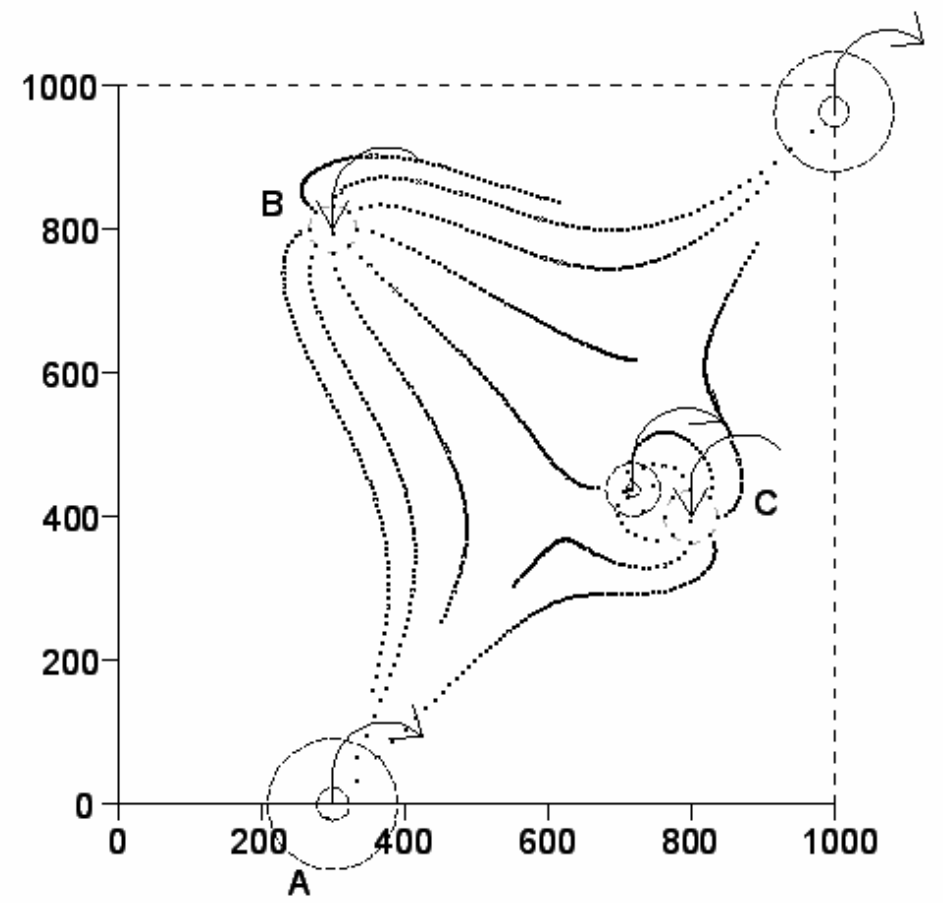

Figure 3. Typical best results (second problem, pattern A) 


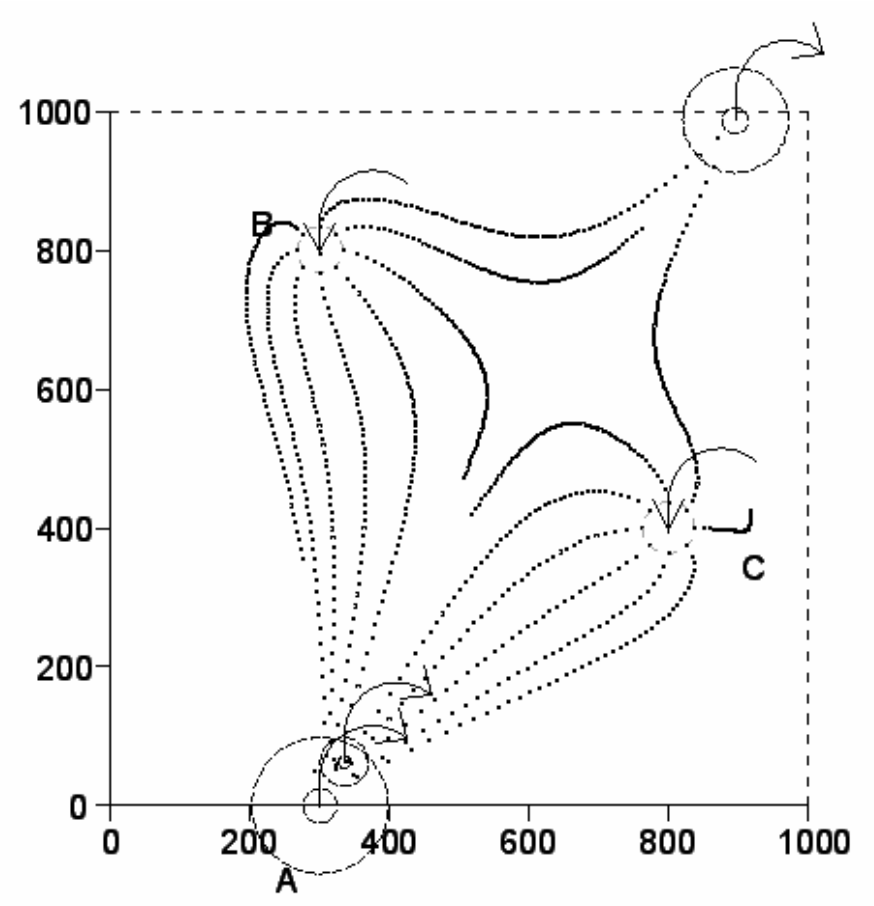

Figure 4. Typical best results (second problem, pattern B)

Table 3. Typical best results for fixed total water flow rate

\begin{tabular}{ccccccc}
\hline Run & $\mathbf{Q}_{\mathbf{A}}$ & $\mathbf{Q}_{\mathrm{D}}$ & $\mathbf{X}_{\mathrm{D}}, \mathbf{Y}_{\mathrm{D}}$ & $\mathbf{Q}_{\mathrm{E}}$ & $\mathbf{X}_{\mathrm{E}}, \mathbf{Y}_{\mathrm{E}}$ & $\mathbf{V B}$ \\
\hline $\mathbf{1}$ & 349.94 & 206.27 & 924,980 & 43.79 & 344,75 & 30630.102 \\
\hline $\mathbf{2}$ & 349.15 & 43.69 & 335,63 & 207.16 & 896,987 & 30231.399 \\
\hline $\mathbf{3}$ & 294.73 & 252.58 & 999,986 & 52.69 & 719,442 & 28973.655 \\
\hline $\mathbf{4}$ & 298.03 & 250.85 & 1000,964 & 51.12 & 719,438 & 28052.425 \\
\hline $\mathbf{5}$ & 269.92 & 83.14 & 810,188 & 246.94 & 1000,1000 & 32266.180 \\
\hline $\mathbf{6}$ & 299.77 & 240.03 & 958,991 & 60.14 & 619,395 & 34566.954 \\
\hline $\mathbf{7}$ & 222.92 & 220.61 & 1000,985 & 156.47 & 998,0 & 31790.796 \\
\hline
\end{tabular}

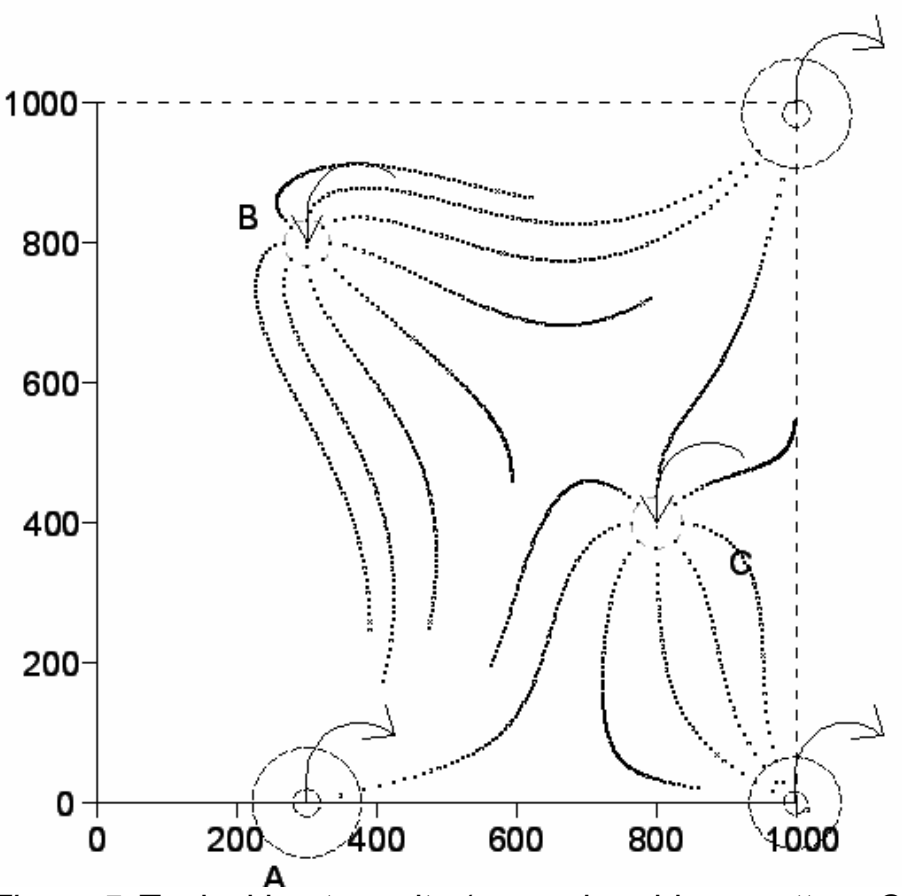

Figure 5. Typical best results (second problem, pattern C) 


\section{DISPERSIVE MASS TRANSPORT}

The simulation model, which has been used in this paper, includes advective mass transport only. A rather "empirical" way to compensate for dispersive mass transport is to increase pollutant deactivation time. Another idea is to follow the approach of Karamagiola and Katsifarakis, (2007), who have studied transport of pollutants, injected in an aquifer at constant rate through a well. They have used a moving point technique, similar to ours, to simulate advective mass transport and they applied the one-dimensional solution of Ogata and Banks (1961) along the path line of each moving point, to take pollutant dispersion into account. The great advantage of this approach is that it increases the total computational volume only slightly, since: a) the required velocity values have already been calculated, in order to simulate advective transport and $b$ ) the $x_{p}$ values (namely the coordinates along each path line) are calculated only once, as the sum of the distances between successive positions of each moving point, up to its examined location. Moreover, the error function is easily calculated with excellent accuracy, using the first 30 terms of its series expansion. The aforementioned approach should be investigated further, though, to estimate whether it is an acceptable substitute of numerical schemes, currently used to simulate advective-dispersive mass transport.

\section{FINAL REMARKS}

Optimizing management of polluted aquifers is not an easy task. The 2 application examples, presented in this paper, show that the proposed combination of genetic algorithms with a moving point code is an efficient tool for such problems. In the first application we seek the maximum total clean water pumping rate $Q_{s}$ from three production wells, situated in the same restricted area with two wells that inject polluted water. Results plausibly show that $Q_{s}$ depends heavily on the dimensions of the available area. In the second application, the total flow rate of the production wells is fixed and the minimum treatment cost of pumped water is sought. Best solutions resulting from a number of runs have been classified in 3 different patterns, but the one which leads to the smallest "cost" values is not the best. This comment implies that results should be carefully evaluated, since simplifications in the flow simulation model, numerical approximations (e.g. in the moving point code) and small inaccuracies in the evaluation function, may misguide the optimization process.

\section{REFERENCES}

Bear J. and Bachmat Y. (1990), Introduction to modeling of transport phenomena in porous media, Springer.

Bedient P.B., Rifai H.S. and Newell C.J. (1994), Ground water contamination transport and remediation, Prentice Hall.

Goldberg D.E. (1989), Genetic algorithms in search, optimization and machine learning, AddisonWesley publishing company, Reading, Massachusetts.

Holland J.H. (1975), Adaptation in natural and artificial systems, University of Michigan Press, Ann Arbor.

Karamagiola E. and Katsifarakis K.L. (2007) An approximate solution to convective-dispersive mass transport in aquifers, In: Proc. CEMEPE 2007, pp 2627-2632.

Katsifarakis K.L. (Ed.) (2000), Groundwater pollution control, WIT Press.

Katsifarakis K.L. and Karpouzos D.K. (1998) Minimization of pumping cost in zoned aquifers by means of genetic algorithms, In: Proc. Int. Conf. "Protection and Restoration of the Environment IV", Katsifarakis K.L., Korfiatis G.P., Mylopoulos Y.A. and Demetracopoulos A.C. (Eds), pp. 61-68.

Katsifarakis K.L., Karpouzos D.K. and Theodossiou N. (1999a), Combined use of BEM and genetic algorithms in groundwater flow and mass transport problems, Engineering Analysis with Boundary Elements, 23(7), 555-565.

Katsifarakis K.L., Latinopoulos P. and Petala Z. (1999b), Application of a BEM and moving point code to the study of pumped coastal aquifers, In: Proceedings BEM 21, C.A. Brebbia C.A. and Power H. (Eds.), WIT Press, pp. 639-647.

Michalewicz Z. (1996), Genetic algorithms + Data structures $=$ Evolution programs, $3^{\text {nd }}$ edition, Springer-Verlag.

Ogata A. and Banks R.B. (1961), A solution of the differential equation of longitudinal dispersion in porous media, Prof. paper 411-A, U.S. Geological Survey. 
Reeves C.R. and Raw J.E. (2003), Genetic algorithms-Principles and perspectives, Kluwer Academic Publishers.

Voudrias E.A. (2001), Pump-and-treat remediation of groundwater contamination by hazardous waste: Can it really be achieved?, Global Nest: The Int. J., 3(1), 1-10. 